\title{
Conhecimento sobre intolerância à lactose entre nutricionistas
}

\author{
Knowledge about lactose intolerance among nutritionists
}

\author{
Andreia Bauermann ${ }^{1}$, Zilda de Albuquerque Santos ${ }^{2}$ \\ ${ }^{1}$ Nutricionista pelo Centro Universitário Metodista IPA, Porto Alegre, RS. \\ 2 Nutricionista. Doutora em Ciências da Saúde pela Pontifícia Universidade Católica do Rio Grande do Sul. Docente da Universidade Federal do \\ Rio Grande do Sul, Porto Alegre, RS.
}

\begin{abstract}
RESUMO
Objetivos: Avaliar o conhecimento de nutricionistas sobre intolerância à lactose e seu manejo nutricional.

Métodos: Foi realizado um estudo transversal prospectivo, com amostra constituída por nutricionistas que atuam na área clínica no Município de Porto Alegre/RS. A coleta dos dados ocorreu através do preenchimento de um questionário auto administrado, composto de questões abertas e fechadas sobre intolerância à lactose e seu tratamento nutricional.

Resultados: Foram avaliados 30 nutricionistas, trabalhando em consultórios ou hospitais, sendo a grande maioria (29) do sexo feminino. A idade variou entre 25 e 58 anos (mediana de 37 anos) e o mínimo tempo de graduação foi de sete anos. Somente um terço dos entrevistados conceituou corretamente intolerância à lactose como presença de sintomas gastrintestinais decorrentes da má absorção de lactose. Em caso de intolerância à lactose em um lactente, $56,5 \%$ dos entrevistados recomendaria manter aleitamento materno e retirar proteínas do leite de vaca da dieta da mãe. Menos da metade dos profissionais (46,6\%) sabiam a dose recomendada de cálcio para adultos entre 19 e 50 anos. Treze entrevistados $(43,3 \%)$ referiram atender frequentemente pacientes com intolerância à lactose. Não houve associação entre melhor conhecimento sobre o manejo da intolerância à lactose e frequência com que os profissionais costumavam atender pacientes com esse distúrbio.
\end{abstract}

Conclusões: A maioria dos nutricionistas desta amostra não estava suficientemente preparada para tratar indivíduos intolerantes à lactose; faltavam conhecimentos adequados sobre essa condição e seu manejo nutricional.

DESCRITORES: INTOLERÂNCIA À LACTOSE; TERAPIA NUTRICIONAL; CÁLCIO NA DIETA; NUTRICIONISTA.

\begin{abstract}
Aims: To evaluate the knowledge of nutritionists about lactose intolerance and its nutritional management.

Methods: A prospective cross-sectional study was conducted, with a sample of nutritionists who work in the clinical area in the city of Porto Alegre, Rio Grande do Sul state, Brazil. Data collection occurred by completing a selfadministered questionnaire consisting of open and closed questions about lactose intolerance and its nutritional treatment.

Results: A total of 30 nutritionists working in hospitals or clinics were interviewed, the vast majority (29) female. The age ranged between 25 and 58 years (median 37 years) and the minimum graduation time was 7 years. Only a third of respondents correctly conceptualized lactose intolerance as gastrointestinal symptoms resulting from lactose malabsorption. In case of lactose intolerance in an infant, $56.5 \%$ of respondents would recommend maintaining breastfeeding and remove cow's milk proteins from the mother's diet. Less than half of professionals $(46.6 \%) \mathrm{knew}$ the recommended dose of calcium for adults aged 19 to 50 years. Thirteen respondents $(43.3 \%)$ reported often meet patients with lactose intolerance. There was no association between better knowledge about the management of lactose intolerance and the frequency with which the professionals used assist patients with this disorder.

Conclusions: Most nutritionists of this sample were not sufficiently prepared to treat individuals with lactose intolerance; they had no adequate knowledge about this condition and its nutritional management.
\end{abstract}

KEY WORDS: LACTOSE; LACTOSE INTOLERANCE; NUTRITIONAL THERAPY; CALCIUM, DIETARY; NUTRITIONIST. 


\section{INTRODUÇÃO}

Lactose é um carboidrato encontrado exclusivamente no leite. É sintetizado nas glândulas mamárias e hidrolisado em glicose e galactose pela enzima lactase, também denominada lactase-florizina hidrolase, no intestino delgado. A presença de manifestações clínicas gastrointestinais decorrentes da má absorção da lactose é denominada intolerância à lactose (IL). Diversas situações podem causar redução na síntese da lactase, com consequente má absorção de lactose. Essas situações são classificadas como deficiência de lactase primária, secundária ou congênita. ${ }^{1,2} \mathrm{~A}$ deficiência primária ocorre quando o organismo reduz, fisiologicamente, a produção de lactase. A condição pode se manifestar na infância, adolescência ou idade adulta, sendo a causa mais comum de IL. A deficiência secundária ocorre como consequência de alguma alteração patológica no intestino delgado, que pode se manifestar em qualquer idade. A deficiência congênita é uma condição grave, rara e irreversível, que se manifesta nos recém-nascidos. ${ }^{1,3,4}$

A prevalência de IL é bastante variada entre a população, sendo menos frequente na raça branca quando comparada com indivíduos de cor negra, parda ou amarela. ${ }^{5-9}$ Aproximadamente $65 \%$ da população humana sofre redução na capacidade de digerir a lactose após a infância e vai apresentar sinais e sintomas ao longo da vida. ${ }^{1}$ Os sintomas típicos que alertam para a suspeita de IL são dor e/ou distensão abdominal, flatulência, diarréia e borborismos (ou borborigmos). ${ }^{10}$ As manifestações clínicas estão diretamente relacionadas com a quantidade de lactose ingerida e com o grau de deficiência na produção da lactase. ${ }^{11}$

A má absorção de lactose pode ser avaliada através do teste do ar hidrogênio expirado, que avalia a expiração pulmonar do gás hidrogênio, produzido por bactérias intestinais, ou a curva glicêmica, que mede o aumento na taxa de glicose; ambos realizados após a ingestão de uma determinada quantidade de lactose. ${ }^{11}$ Outros exames utilizados são ${ }^{13} \mathrm{C}$-Lactose para complementar o teste do hidrogênio expirado, avaliação da atividade da lactase em biópsia jejunal, teste para defeitos genéticos, medida de $\mathrm{pH}$ fecal, medida de galactose urinária e o registro dos sintomas após a ingestão de uma dose de lactose, chamado teste desafio. $^{12}$

O tratamento da IL depende da origem da má absorção deste carboidrato. Na deficiência congênita, o tratamento consiste em remover totalmente a lactose da dieta. ${ }^{13} \mathrm{Na}$ deficiência secundária, o tratamento deve ser direcionado para a doença de base. ${ }^{14} \mathrm{Na}$ deficiência primária, inicialmente é preciso identificar a quantidade de lactose tolerada (aquela em que o indivíduo não apresenta sintomas, ou estes não são significativos) e quais produtos lácteos podem ser tolerados (leites, queijos e iogurtes); a partir dessas informações prescreve-se a dieta, que é a base do tratamento. A orientação básica consiste na prescrição de alimentos lácteos de acordo com a tolerância do paciente e reposição adequada de nutrientes que possam ter sido excluídos ou reduzidos, como por exemplo o cálcio. Por fim, e se necessário, pode ser prescrito o tratamento medicamentoso..$^{15,16}$

Considerando que a deficiência primária de lactase é bastante comum na população, é esperado que uma importante parcela destes indivíduos desenvolva IL. Sendo o manejo clínico desta situação eminentemente nutricional, o nutricionista passa a ser um profissional fundamental no tratamento; por isso, seu conhecimento acerca das possibilidades de adaptações nutricionais necessárias na alimentação desses indivíduos é fundamental. Assim, o objetivo deste estudo foi avaliar o conhecimento dos nutricionistas sobre IL e seu manejo nutricional.

\section{MÉTODOS}

Foi realizado um estudo transversal analítico, envolvendo uma amostra de nutricionistas que atuavam na área clínica, trabalhando em hospitais ou consultórios, no Município de Porto Alegre/RS, no período de janeiro a fevereiro de 2012. O cálculo do tamanho da amostra, em 30 participantes, foi feito com base no estudo de Cortez et al. ${ }^{18} \mathrm{O}$ estudo foi aprovado pelo Comitê de Ética em Pesquisa do Centro Universitário Metodista do IPA, sob o protocolo número 328/2011. Todos os participantes assinaram o Termo de Consentimento Livre e Esclarecido.

Os nutricionistas participantes do estudo foram selecionados inicialmente entre conhecidos das autoras, independentemente de atuarem em hospitais, consultórios ou postos de saúde. Todos foram contatados, aleatoriamente, pessoalmente ou por telefone celular, sendo informados sobre os objetivos do estudo e convidados a participar da pesquisa. Todos os contatos foram realizados em horário comercial e os que não atenderam à chamada telefônica retornaram a ligação em um breve período de tempo. Os profissionais que aceitaram participar também indicaram colegas, que foram então contatados por e-mail, no qual seguia uma breve apresentação do estudo, seus objetivos e a solicitação de retorno dos que aceitassem participar. Os contatos foram feitos até que se obtivessem 
os 30 profissionais que aceitaram participar do estudo. As pesquisadoras desconheciam a experiência dos participantes no manejo da IL e nenhum deles era especialista na área de gastroenterologia. $\mathrm{O}$ critério de seleção foi atuar na área de Nutrição Clínica.

No momento do contato, foi agendado um horário para aplicação de um questionário e assinatura do termo de consentimento. O questionário, elaborado pelas pesquisadoras, era dividido em duas partes: uma parte inicial buscando informações sobre idade, sexo, tempo de graduação, grau de especialização e uma autoavaliação sobre o conhecimento a respeito da IL; e uma segunda parte composta por perguntas de múltipla escolha sobre conhecimentos gerais e conduta na IL. A segunda parte foi elaborada e analisada com base na fisiopatologia e manejo da IL, descritos na literatura. $\mathrm{O}$ respondente não era identificado no documento.

Os resultados foram organizados em um banco de dados do programa Excel e a análise dos dados utilizou o software SPSS (Statistical Package for the Social Sciences) versão 17.0. As variáveis quantitativas foram descritas através de percentual e as variáveis categóricas foram descritas através de frequências absolutas e relativas. Para comparar médias entre os grupos foi aplicada a Análise de Variância (ANOVA) one-way com post-hoc de Tukey. Para comparar proporções entre os grupos, foi aplicado o teste qui-quadrado de Pearson. O nível de significância estatística considerado foi de $5 \%(\mathrm{p}<0,05)$.

\section{RESULTADOS}

Entre os 30 profissionais avaliados, 29 (96,6\%) eram do sexo feminino. A idade mínima e máxima da amostra ficou entre 25 e 58 anos, com mediana de 37 anos. A maioria tinha entre 30-39 anos de idade (40\%), seguidos por aqueles entre 40-49 anos $(26,66 \%)$, $25-29$ anos $(23,3 \%)$ e $50-58$ anos $(10 \%)$. O tempo de formatura era no mínimo de sete anos, apresentavam especialização em diferentes áreas da nutrição e trabalhavam em consultório ou hospitais. Treze profissionais $(43,4 \%)$ referiram atender frequentemente pacientes com IL e 76,6\% consideravam suficientes seus conhecimentos sobre essa condição clínica (Tabela 1).

A Tabela 2 apresenta os resultados das variáveis relacionadas ao conhecimento e conduta dos nutricionistas sobre IL. Pode-se observar que um terço dos pesquisados conceituaram corretamente IL como presença de sintomas gastrintestinais decorrentes da má absorção de lactose.
Ao se investigar se o conhecimento técnico sobre a IL era associado com a frequência com que o profissional nutricionista atendia pacientes com esse distúrbio, não foi encontrada associação significativa.

Tabela 1. Perfil da amostra de 30 nutricionistas que atuavam na área clínica em Porto Alegre, estado do Rio Grande do Sul, no período de janeiro a fevereiro de 2012, e autoavaliação dos mesmos quanto aos conhecimentos sobre intolerância à lactose.

\begin{tabular}{|c|c|}
\hline Variáveis & n $(\%)$ \\
\hline \multicolumn{2}{|l|}{ Sexo } \\
\hline Feminino & $29(96,6)$ \\
\hline Masculino & $1 \quad(3,3)$ \\
\hline \multicolumn{2}{|l|}{ Idade } \\
\hline 25 a 29 anos & $7(23,3)$ \\
\hline 30 a 39 anos & $12(40,0)$ \\
\hline 40 a 49 anos & $8(26,6)$ \\
\hline 50 a 58 anos & $3(10,0)$ \\
\hline \multicolumn{2}{|l|}{ Local de trabalho } \\
\hline Consultório & $18(60,0)$ \\
\hline Posto de saúde & $2(6,6)$ \\
\hline Outro local & $10(33,3)$ \\
\hline \multicolumn{2}{|l|}{ Tempo de graduação } \\
\hline Mais de 7 anos & $20(66,6)$ \\
\hline Menos de 7 anos & $10(33,4)$ \\
\hline \multicolumn{2}{|l|}{ Pós-graduação } \\
\hline Não possui & $1(3,3)$ \\
\hline Especialização & $20(66,6)$ \\
\hline Mestrado & $3(10,0)$ \\
\hline Doutorado & $2(6,6)$ \\
\hline Especialização e mestrado & $4(13,3)$ \\
\hline \multicolumn{2}{|c|}{$\begin{array}{l}\text { Frequência com que atende pacientes com } \\
\text { intolerância à lactose }\end{array}$} \\
\hline Frequentemente & $13(43,3)$ \\
\hline Ocasionalmente & $11(36,6)$ \\
\hline Raramente & $6(20,0)$ \\
\hline \multicolumn{2}{|c|}{$\begin{array}{l}\text { Autoavaliação quanto ao nível de conhecimento } \\
\text { sobre intolerância à lactose }\end{array}$} \\
\hline Ótimo & $1(3,3)$ \\
\hline Suficiente & $23(76,6)$ \\
\hline Insuficiente & $6(20,0)$ \\
\hline
\end{tabular}


Tabela 2. Conhecimentos sobre intolerância à lactose por parte de 30 nutricionistas que atuavam na área clínica em Porto Alegre, estado do Rio Grande do Sul, no período de janeiro a fevereiro de 2012 (n=30).

\section{Questão}

O conceito de intolerância à lactose é

o resultado fisiológico da baixa produção de lactase

o resultado da má absorção de lactose

a presença de sintomas gastrintestinais decorrentes da má absorção de lactose

$10(33,3)$

São sintomas típicos de intolerância à lactose

diarréia e distensão abdominal

flatulência

borborismos

$14(46,6)$

constipação

$11(36,6)$

náuseas e vômitos

$6(26,6)$

Ao atender um paciente com intolerância à lactose:

sugere exclusão do leite de vaca

orienta reduzir a ingestão de leite e produtos lácteos

sugere substitutos do leite de vaca

prescreve enzimas digestivas

$10(33,3)$

Alimentos que são indicados no manejo da intolerância à lactose:

leite de soja

leite de arroz

leite de cabra

iogurte com cepas probióticas

$10(33,3)$

iogurte sem cepas probióticas

leite fermentado

$6(20,0)$

leite com baixo teor de lactose

queijos curados

$8(26,6)$

suco de soja

Como orienta a ingestão diária de cálcio

a partir de outros alimentos

suplementação

não se preocupa com a ingestão de cálcio no primeiro momento

Realiza cálculo de ingestão de cálcio para estes pacientes

Não realiza cálculo de ingestão de cálcio para estes pacientes

A recomendação de ingestão adequada de cálcio em adultos entre 19 e 50 anos é:

$1200 \mathrm{mg}$

$1100 \mathrm{mg}$

$1000 \mathrm{mg}$

$14(46,6)$

$800 \mathrm{mg}$

$8(26,6)$

Faz orientação sobre a leitura de rótulos de produtos industrializados

Não faz orientação sobre a leitura de rótulos de produtos industrializados

O que deve ser observado na leitura de rótulo:

leite e frações, lacto-albumina, caseína, queijo e manteiga

apenas a presença de leite

outro tipo de orientação deve ser dada

$5(16,6)$

Em lactentes com intolerância à lactose deve-se:

manter aleitamento e retirar proteínas do leite de vaca da dieta da mãe

$17(56,5)$

prescrever fórmulas em substituição ao leite materno

(não atendiam lactentes)

O que deve ser excluído da dieta de intolerantes à lactose:

lactose

proteínas do leite de vaca e lactose 


\section{DISCUSSÃO}

Escolher alimentos sem lactose ou que possuam pouca quantidade da mesma produz remissão dos sintomas dos indivíduos com IL, influenciando positivamente a qualidade de vida desses pacientes. ${ }^{18-20}$ Assim, o nutricionista é um profissional importante no manejo dessa intolerância alimentar. Apesar disso, não encontramos estudos que avaliaram o conhecimento deste profissional sobre os seus aspectos clínicos e dietoterápicos. Em nossa amostra observamos que predominou o sexo feminino, concordando com Archanjo e Brito ${ }^{17}$ que, ao descreverem o perfil do profissional nutricionista brasileiro, ressaltam ser esta uma profissão na qual predominam as mulheres.

Algumas doenças, como alergia alimentar e IL, possuem na alimentação a base do seu tratamento. Sendo assim, espera-se que o profissional nutricionista domine o manejo dessas condições. Cortez et al., ${ }^{18}$ ao avaliarem o conhecimento técnico de 29 nutricionistas no manejo da alergia alimentar, encontraram que $59 \%$ estavam capacitados para orientar o tratamento não medicamentoso. Resultado diferente do encontrado em nosso estudo, que mostrou que menos da metade da amostra apresentou conhecimento satisfatório para tratar indivíduos intolerantes à lactose, apesar de a grande maioria afirmar apresentar conhecimento suficiente.

Define-se como IL a presença de sintomas gastrintestinais decorrentes da má absorção de lactose. ${ }^{19,20}$ A maior parte dos participantes demonstrou não conhecer esta definição. As manifestações clínicas assinaladas como as mais frequentes e que sugerem IL foram diarreia, flatulência, distensão abdominal; o sintoma de borborismos (ou borborigmos), apesar de pouco assinalado, também faz parte do quadro clínico. No entanto, constipação, náuseas e vômito são considerados sintomas atípicos..$^{21-23}$

No curso da IL, muitas vezes os sintomas típicos, por sua intensidade ou frequência, são incompatíveis com uma boa qualidade de vida. Para minimizá-los, é necessária uma dieta de exclusão e/ou restritiva, o que pode resultar em adesão parcial ou inadequada por parte dos pacientes. Na tentativa de minimizar restrições alimentares, os gastroenterologistas prescrevem a enzima lactase para uso em situações especiais ou eventuais. Foi referida por $33,3 \%$ dos profissionais participantes deste estudo a prescrição de enzimas digestivas; no entanto, o Conselho Federal de Nutricionistas diz que lactase não é um alimento, logo não é da competência do nutricionista prescrever. ${ }^{24}$ Reforça esta recomendação o fato de a Agência Nacional de Vigilância Sanitária (ANVISA) incluir enzimas digestivas na categoria de medicamentos, os quais devem ser indicados somente por prescrição médica. ${ }^{25}$

Embora a maioria dos participantes do estudo tenha afirmado calcular a ingestão de cálcio dos seus pacientes, menos da metade dos pesquisados souberam qual é a ingestão diária recomendada (Recommended Dietary Allowance, RDA) desse mineral, que é de $1000 \mathrm{mg} /$ dia para indivíduos entre 19 e 50 anos. ${ }^{26}$ A redução na ingestão de produtos lácteos restringe concomitantemente a ingestão de cálcio, mas felizmente diversos produtos enriquecidos com esse nutriente estão disponíveis à população e são substitutos indicados por diversos autores. ${ }^{27-29}$ No entanto, poucos profissionais citaram esses produtos como alternativas alimentares, alguns preferindo utilizar suplemento como fonte complementar de cálcio.

Segundo Montalto et al., ${ }^{30}$ ao orientar uma dieta o nutricionista deve também ensinar o paciente a ler e interpretar rótulos de produtos industrializados. Em nosso estudo esta foi uma prática referida por quase todos os profissionais, porém a maioria indicou orientar a exclusão de alimentos que contenham em seus rótulos nutrientes não restritos para intolerantes à lactose, como lacto-albumina, caseína e manteiga, limitando ainda mais a dieta dos pacientes.

Em 1999, Host et al. publicaram que lactentes com IL precisam de fórmulas específicas para sua alimentação. ${ }^{31}$ A Academia Americana de Pediatria (2006) ${ }^{32}$ também destacou a importância de substituir o leite materno, pois mesmo uma dieta materna restrita em lactose não isenta o leite materno desse carboidrato. Nossos resultados mostram que os profissionais ficaram em dúvida quanto a essa prática, uma vez que metade da amostra orientaria o uso de fórmulas e a outra metade faria restrição de proteínas do leite de vaca na dieta materna. Isso alerta para a confusão que parece existir entre IL e alergia à proteína do leite de vaca. Embora, quando questionados pontualmente sobre o que excluir na dieta de intolerantes à lactose, o grupo referiu ser necessário excluir apenas a lactose.

Nossos resultados apontam que o profissional nutricionista desta amostra, ainda não está suficientemente preparado para tratar indivíduos intolerantes à lactose. Faltou um conhecimento mais técnico sobre a doença e, sobretudo, sobre as possibilidades de conduta nutricional.

\section{AGRADECIMENTOS}

Aos nutricionistas que participaram deste estudo. 


\section{REFERÊNCIAS}

1. Heyman SR. Lactose intolerance in infants, children, and adolescents. Pediatrics. 2006;118(3):1279-86.

2. Vesa TH, Marteau P, Korpela R. Lactose intolerance. Am J Coll Nutr. 2000;19(2 Suppl):165S-175S.

3. Madry E, Lisowska A, Kwiecien J, Marciniak R, KorzonBurakowska A, Drzymała-Czyż S, et al. Adult-type hypolactasia and lactose malabsorption in Poland. Acta Biochim Pol. 2010;57(4):585-8.

4. United States National Library of Medicine. Genetics Home Reference. Lactose intolerance. [monografia na internet]. Bethesda (MD): NLM; 2011. [capturado em 2011 out 17]. Disponível em: http://ghr.nlm.nih.gov/condition/lactoseintolerance

5. Babu J, Kumar S, Babu P, Prasad JH, Ghoshal UC. Frequency of lactose malabsorption among healthy southern and northern Indian populations by genetic analysis and lactose hydrogen breath and tolerance rests. Am J Clin Nutr. 2010;91(1):140-6.

6. Malmström H, Linderholm A, Lidén K, Storå J, Molnar P, Holmlund G, et al. High frequency of lactose intolerance in a prehistoric Hunter-gatherer population in northern Europe. BMC Evol Biol. 2010;10:89.

7. Mattar R, Monteiro MS, Villares CA. Frequency of LCT$13810 \mathrm{C}>\mathrm{T}$ single nucleotide polymorphism associated with adult-type hypolactasia/lactase persistence among Brazilians of different ethnic groups. Nutr J. 2009;8:46.

8. Tishkoff SA, Reed FA, Ranciaro A, Voight BF, Babbitt CC, Silverman JS, et al. Convergent adaptation of human lactase persistence in Africa and Europe. Nat Genet. 2007;39(1): 31-40.

9. Lokki AI, Järvelä I, Israelsson E, Maiga B, Troye-Blomberg M, Dolo A, et al. Lactase persistence genotypes and malaria susceptibility in Fulani of Mali. Malar J. 2011;10:9.

10. Adimora GN, Ikefuna AN, Ilechukwu G. Home management of childhood diarrhoea: need to intensify campaign. Niger J Clin Pract. 2011;14(2):237-41.

11. Jellema P, Schellevis FG, Van der Windt DAWM, Kneepkens CMF, Van der Horst HE. Lactose malabsorption and intolerance: a systematic review on the diagnostic value of gastrointestinal. QJM. 2010;103(8):555-72.

12. Hovde O, Farup PG. A comparison of diagnostic tests for lactose malabsorption: which one is the best? BMC Gastroenterol. 2009;9:82.

13. Lomer MCE, Parkes GC, Sanderson JD. Review article: lactose intolerance in clinical practice - myths and realities. Aliment Pharmacol Ther. 2008;27(2):93-103.

14. Ingram CJE, Mulcare CA, Itan Y, Thomas M, Swallow DM. Lactose digestion and the evolutionary genetics of lactase persistence. Hum Genet. 2009;124(6):579-91.

15. Matthews SB, Waus JP, Roberts AG, Campbell AK. Systemic lactose intolerance: a new perspective on an old problem. Postgrad Med J. 2005; 81(953):167-73.

16. Montalto M, Curigliano V, Santoro L, Vastola M, Cammarota G, Manna R, et al. Management and treatment of lactose malabsorption. World J Gastroenterol. 2006;12(2):187-91.

17. Archanjo LR, Brito KFW. Nutrição: gênero e profissão. RUBS. 2005;1(4):44-50.
18. Cortez APB, Medeiros LCS, Speridião PGL, Mattar RHGM, Neto UF, et al. Conhecimento de pediatras e nutricionistas sobre o tratamento da alergia ao leite de vaca no lactente. Rev Paul Pediatr. 2007;25(2):106-13.

19. Suarez FL, Savaiano DA, Levitt MD. A comparison of symptoms after the consumption of milk or lactosehydrolyzed milk people with self-reported severe lactose intolerance. N Engl J Med. 1995;333(1):1-4.

20. Ehrenpreis ED, Ehrenpreis BZ. Lactose intolerance: definition, symptoms and treatment [monografia na internet]. Milwaukee (WI): IFFGD; 2007. [capturado em 19 out 2011]. Disponível em: http:/www.iffgd.org/store/ viewproduct/122

21. National Institutes of Health. NIH Consensus Development Conference: Lactose Intolerance and Health; 2010 Feb 22 24; Bethesda (MD). Kensington, MD: AHRQ; 2010.

22. Martini MC, Savaiano DA. Reduced intolerance symptoms from lactose consumed during a meal. Am J Clin Nutr. 1988;47(1):57-60.

23. Lovelace, HY, Barr SI. Diagnosis, smptoms, and calcium intakes of individuals with self-reported lactose intolerance. J Am Coll Nutr. 2005;24(1):51-7.

24. Brasil. Conselho Federal de Nutricionistas. Resolução $n^{0}$ 380 de 2005. Brasília (DF); 2005. [capturado em 2011 out 01]. Disponível em: http://www.cfn.org.br/novosite/pdf/ res/2005/res380.pdf

25. Brasil. Ministério da Saúde. Agência Nacional de Vigilância Sanitária. Alimentos com alegações de propriedades funcionais e ou de saúde. Brasília (DF); 2005 [capturado em 2011 set 02]. Disponível em: http://portal.anvisa.gov.br

26. Institute of Medicine, Food and Nutrition Board. Dietary Reference Intakes: recommended intakes for individuals. Washington (DC); 2004 [capturado em 2011 out 03]. Disponível em: http://fnic.nal.usda.gov/dietary-guidance/ dietary-reference-intakes

27. Grundmann SA, Stratmann E, Brehler R, Luger TA, Ständer S. Lactase deficiency: a potential novel aetiological factor in chronic pruritus of unknown origin. Acta Derm Venereol. 2011;91(6):698-703.

28. Lember M, Torniainen S, Kull M, Kallikorm R, Saadla $\mathrm{P}$, Rajasalu $\mathrm{T}$, et al. Lactase non-persistense and milk consumption in Estonia. World J Gastroenterol. 2006; 12(45):7329-31.

29. Savaiano DA, AbouElAnour A, Smith DE, Levitt MD. Lactose malabsorption from yogurt, pasteurized yogurt, sweet acidophilus milk, and cultured milk in lactasedeficient individuals. Am J Clin Nutr. 1984;40(6):1219-23.

30. Montalto M, Gallo A, Santoro L, D'Onofrio F, Curigliano V, Covino M, et al. Low-dose lactose in drugs neither increases breath hydrogen excretion nor causes gastrointestinal symptoms. Aliment Pharmacol Ther. 2008;28(8):1003-12.

31. Host A, Koletzko B, Dreborg S, Muraro A, Wahn U, Aggett $\mathrm{P}$, et al. Dietary products used in infants for treatment and prevention of food allergy. Arch Dis Child. 1999;81(1):80-4.

32. Green M, Palfrey JS, editors. Bright futures: guidelines for health supervision of infants, children, and adolescents

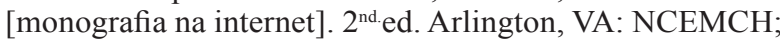
2002 [capturado em 20115 Set 03]. Disponível em: http:// www.brightfutures.org/bf $2 / \mathrm{pdf}$ 